Correction

\title{
Correction: Co-targeting translation and proteasome rapidly kills colon cancer cells with mutant RAS/RAF via ER stress
}

\section{Xiangyun Li ${ }^{1,3, *}$, Mei Li ${ }^{2,3, *}$, Hang Ruan ${ }^{3}$, Wei Qiu ${ }^{1}$, Xiang $X \mathbf{u}^{1}$, Lin Zhang ${ }^{4}$, Jian $\mathbf{Y u}^{3}$}

${ }^{1}$ First department, State Key Laboratory of Trauma, Burn and Combined Injury, Research Institute of Surgery and Daping Hospital, Third Military Medical University, Daping, Yu Zhong District, Chongqing 400042, P.R. China

2 Department of Animal Genetics, Breeding and Reproduction, Nanjing Agricultural University, Weigang, Nanjing 210095, P.R. China

${ }^{3}$ Department of Pathology, University of Pittsburgh Cancer Institute, Pittsburgh, PA 15213, USA

${ }^{4}$ Department of Pharmacology and Chemical Biology, University of Pittsburgh School of Medicine, University of Pittsburgh Cancer Institute, Pittsburgh, PA 15213, USA

* These authors contributed equally to this work

Correspondence to: Mei Li, email: limei@njau.edu.cn

Jian Yu, email: yuj2@upmc.edu

Xiang Xu, email: xiangxu@ymail.com

Published: January 22, 2018

Copyright: Li et al. This is an open-access article distributed under the terms of the Creative Commons Attribution License 3.0 (CC BY 3.0), which permits unrestricted use, distribution, and reproduction in any medium, provided the original author and source are credited.

This article has been corrected: Dr. Mei Li is now listed as the first correspondence author instead of Dr. Jian Yu in the correspondence section.

Original article: Oncotarget. 2017; 8:9280-9292. https://doi.org/10.18632/oncotarget.14063 